\title{
Variationally Universal Hashing
}

\author{
Ted Krovetz $^{\mathrm{a}}$ and Phillip Rogaway ${ }^{\mathrm{b}, \mathrm{c}}$ \\ ${ }^{a}$ Department of Computer Science, California State University \\ Sacramento CA 95819 USA \\ ${ }^{\mathrm{b}}$ Department of Computer Science, University of California \\ Davis CA 95616 USA \\ ${ }^{\mathrm{c}}$ Department of Computer Science, Faculty of Science, Chiang Mai University \\ Chiang Mai 50200 Thailand
}

\begin{abstract}
The strongest well-known measure for the quality of a universal hash-function family $H$ is its being $\varepsilon$-strongly universal, which measures, for randomly chosen $h \in H$, one's inability to guess $h\left(m^{\prime}\right)$ even if $h(m)$ is known for some $m \neq m^{\prime}$. We give example applications in which this measure is too weak, and we introduce a stronger measure for the quality of a hash-function family, $\varepsilon$-variationally universal, which measures one's inability to distinguish $h\left(\mathrm{~m}^{\prime}\right)$ from a random value even if $h(m)$ is known for some $m \neq m^{\prime}$. We explain the utility of this notion and provide an approach for constructing efficiently computable $\varepsilon$-VU hash-function families.
\end{abstract}

Key words: Randomized algorithms, cryptography, hashing, universal hashing.

\section{Background}

A hash-function family $H=\{h: A \rightarrow B\}$ is a collection of hash functions, each $h \in H$ having the same domain $A$ and codomain $B$, with $B$ finite. One assumes a hash-function family to be samplable: one can choose a random $h$ from $H$. Carter and Wegman introduced hash-function families, and they and Stinson give various measures of their quality [3,6,7], as we now describe.

Hash-function family $H$ is strongly universal (SU) if for all distinct values $m, m^{\prime}$ from the domain, the pair $\left(h(m), h\left(m^{\prime}\right)\right)$ is uniformly distributed when $h$ is randomly sampled from $H$. Two relaxations of SU are $\varepsilon$-almost universal ( $\varepsilon$-AU) and $\varepsilon$-strongly universal $(\varepsilon-\mathrm{SU})$, where $0 \leq \varepsilon \leq 1$ is a real number. Hash-function family $H=\{h: A \rightarrow B\}$ is $\varepsilon$-AU if the probability that any two distinct values $m, m^{\prime}$ collide (ie, hash to the same output) when hashed 
by a randomly selected member of $H$ is at most $\varepsilon$. When $\varepsilon$ is deemed small we say, informally, that $H$ is almost-AU. A hash-function family $H$ is $\varepsilon$-SU if for all distinct $m, m^{\prime}$ from domain $A$ and all $c, c^{\prime}$ from codomain $B$,

(1) $\operatorname{Pr}_{h \in H}[h(m)=c]=\frac{1}{|B|}$, and

(2) $\operatorname{Pr}_{h \in H}\left[h\left(m^{\prime}\right)=c^{\prime} \mid h(m)=c\right] \leq \varepsilon$.

The first condition says that $h(m)$ is uniformly distributed over $B$ and the second condition says that you cannot guess $h\left(m^{\prime}\right)$ with probability better than $\varepsilon$ even if you know $h(m)$. When $\varepsilon$ is deemed small we say, informally, that $H$ is almost-SU.

Almost-AU and almost-SU hash functions have proven to be useful tools. Constructions and software implementations have been given for $\varepsilon$-AU and $\varepsilon$-SU hash-function families with small $\varepsilon$, say $\varepsilon \leq 2^{-30}$, and peak processing rates of less than one CPU cycle per byte of data being hashed $[1,2,4]$. Known constructions for SU families are much slower to compute.

While the notion of an almost-SU hash-function family might seem strong, we suggest that it is weaker than one may need to be generally useful. As an example, fix a nonempty set $A$ and consider the hash-function family $H^{*}=$ $\left\{h_{f, c}: A \rightarrow\{0,1\}^{128}\right\}$ where $f: A \rightarrow\{0,1\}^{64}$ is a function and $c$ is a 64 -bit string. Let $h_{f, c}(x)=f(x) \| c$. Choosing a random member of $H^{*}$ is achieved by uniformly selecting a value $c$ from $\{0,1\}^{64}$ and, for each $x \in A$, assigning to $f(x)$ a uniformly selected value from $\{0,1\}^{64}$. Then $H^{*}$ is $2^{-64}$-SU, which sounds good, but there are natural applications where it is less appropriate than one might expect. For example, consider a hash table of $2^{10}$ entries where each element $x \in A$ is mapped to a position in the table by using as an index the last 10 bits of $h(x)$. Here we randomly choose $h \in H^{*}$ before we begin to hash. Since $H^{*}$ is $2^{-64}$-SU and our table has only $2^{10}$ entries, one might think that $H^{*}$ should work fine for this application. But clearly it will not, hashing all values to the same table entry. Truncated Wegman-Carter message authentication makes for another natural example. If one uses $H^{*}$ to make a Wegman-Carter message authentication code [7], xoring hash outputs with a random string, and then, for concision, truncating the result to the final 64-bits, then all security is lost, since all messages produce the same result.

Although $H^{*}$ is contrived, the examples are not, and they suggest that the definition of $\varepsilon$-SU, which focuses on one's inability to know the entire value of $h\left(m^{\prime}\right)$ once $h(m)$ is known, may not be a technically desirable way to relax the definition of SU when hash outputs undergo further processing. One should instead capture the idea that everything about $h\left(m^{\prime}\right)$ looks random, even if one knows $h(m)$. (In particular, the last 10 or 64 bits will look random.) This paper formalizes this notion and gives an efficient construction meeting it, thus creating a more generally useful class of hash functions for applications. 


\section{Almost-VU Hash Functions}

First we recall a standard notion for the distance between two probability distributions. If $X$ is a random variable over set $S$ with distribution $D$ and probability mass function $p(x)=\operatorname{Pr}[X=x]$, and $X^{\prime}$, also over $S$, has distribution $D^{\prime}$ and mass function $p^{\prime}(x)=\operatorname{Pr}\left[X^{\prime}=x\right]$, then the variational distance between $D$ and $D^{\prime}$ is

$$
\operatorname{dist}\left(D, D^{\prime}\right)=\sum_{\substack{y \in S \\ p(y)>p^{\prime}(y)}}\left(p(y)-p^{\prime}(y)\right)=\frac{1}{2} \sum_{y \in S}\left|p(y)-p^{\prime}(y)\right| .
$$

For finite $S$, let Uniform $(S)$ be the uniform distribution over $S$. When $D^{\prime}$ is $\operatorname{Uniform}(S)$ then $p(y)-p^{\prime}(y)$ in this distance measure is $p(y)-1 /|S|$.

Definition of $\varepsilon$-VU. We suggest strengthening the definition of $\varepsilon$-SU by measuring the variational distance, given knowledge of $h(m)$, between the distribution of $h\left(\mathrm{~m}^{\prime}\right)$ and the uniform distribution. We say that hash-function family $H=\{h: A \rightarrow B\}$ is $\varepsilon$-variationally universal ( $\varepsilon-\mathrm{VU})$ if for all distinct $m, m^{\prime} \in A$, and all $c \in B$,

(1) $\operatorname{Pr}_{h \in H}[h(m)=c]=\frac{1}{|B|}$, and

(2) $\frac{1}{2} \sum_{y \in B}\left|\operatorname{Pr}_{h \in H}\left[h\left(m^{\prime}\right)=y \mid h(m)=c\right]-\frac{1}{|B|}\right| \leq \varepsilon$.

The first condition again says that $h(m)$ is uniformly distributed over $B$ while the second condition says the variational distance between Uniform $(B)$ and the distribution induced on $h\left(m^{\prime}\right)$ when $h(m)=c$ is no more than $\varepsilon$. In other words, we demand that for any distinct $m$ and $m^{\prime}$, the value $h\left(m^{\prime}\right)$ should look uniform even if we know $h(m)$. The quantity $\varepsilon$ measures how far from uniform $h\left(m^{\prime}\right)$ might be. If $\varepsilon$ is deemed small we may say, informally, that $H$ is almost-VU.

With regard to the motivating examples, the $\varepsilon$-VU definition ensures good properties when outputs are truncated. It is not hard to show that if $H$ is an $\varepsilon$-VU hash-function family with each function returning $n$-bit strings, then returning the strings truncated to $m$ bits (any $1 \leq m \leq n$ bits may be selected) yields a hash-function family that is still $\varepsilon$-VU. This means it is always safe to truncate bits produced by an almost-VU hash-function family whereas this is not always the case with an almost-SU one.

An EQUivalent Formulation. Another natural way to claim that a hash function appears random over two points is to say that no algorithm can do well at distinguishing between the hash-values of two distinct inputs and a random pair of codomain points. A hash-function family $H=\{h: A \rightarrow B\}$ 
would be deemed $\varepsilon$-good under this notion if $h(m)$ is uniform for any $m \in A$, as before, and for all functions $f: B^{2} \rightarrow\{0,1\}$, for all distinct inputs $m, m^{\prime} \in A$, we have $\operatorname{Pr}_{h \in H}\left[f\left(h(m), h\left(m^{\prime}\right)\right)=1\right]-\operatorname{Pr}_{x, y \in B}[f(x, y)=1] \leq \varepsilon$. This notion is weaker than our $\varepsilon$-VU definition because the function $f$ has no control of the value $h(m)$ when analyzing the output of $h\left(m^{\prime}\right)$. In contrast, the following definition allows the value for $h(m)$ to be arbitrarily chosen and is equivalent to our definition of $\varepsilon$-VU. Hash-function family $H=\{h: A \rightarrow B\}$ is $\varepsilon$-VU if for all functions $f: B \rightarrow\{0,1\}$, for all distinct $m, m^{\prime} \in A$, for all $c \in B$,

$$
\begin{aligned}
& \operatorname{Pr}_{h \in H}[h(m)=c]=\frac{1}{|B|}, \text { and } \\
& \operatorname{Pr}_{h \in H}\left[f\left(h\left(m^{\prime}\right)\right)=1 \mid h(m)=c\right]-\operatorname{Pr}_{b \in B}[f(b)=1 \mid h(m)=c] \leq \varepsilon
\end{aligned}
$$

The difference of inequality (2) is maximized when $f$ is the function that returns 1 only on values $y \in B$ for which $\operatorname{Pr}_{h \in H}\left[h\left(m^{\prime}\right)=y \mid h(m)=c\right]>$ $1 /|B|$. When this is the case, computing the difference is identical to computing the variational distance between $\operatorname{Uniform}(B)$ and the distribution induced on $h\left(m^{\prime}\right)$ when $h(m)=c$. This indicates that this definition is equivalent to our original formulation of $\varepsilon$-VU.

Almost-SU is WeAKer than ALmost-VU. Any almost-VU family of hash functions is almost-SU as well; specifically, if $H$ is an $\varepsilon$-VU hash-function family with codomain $B$ then it is also $(\varepsilon+1 /|B|)$-SU. The converse is not true. Think back to hash-function family $H^{*}$ described in Section 1. It is almost$\mathrm{SU}$, but it is not almost-VU. This hash-function family satisfies part (1) of the $\varepsilon$-VU definition but it only satisfies part (2) for high $\varepsilon$. For each randomly chosen $h \in H^{*}$ there are only $2^{64}$ strings that can be produced because $h$ always produces the same trailing 64 bits, and for each input all $2^{64}$ possible outputs are equiprobable. So the distance between the distribution for $h(m)$ and Uniform $\left(\{0,1\}^{128}\right)$ is $2^{64}\left(2^{-64}-2^{-128}\right)=1-2^{-64}$.

Are typical ALmost-SU CONSTRUCtions ALmost-VU? The degenerate example above notwithstanding, one might wonder if well-known constructions for almost-SU hash functions are already almost-VU. Certainly SU hash-function families are 0-VU, but typical constructions for almost-SU hash functions will not be almost-VU. As an example, consider hashing using polynomial evaluation $[1,3,5]$. In one form of this paradigm, inputs are broken up into words and the words are interpreted as coefficients in a polynomial over some finite field, say the field with $p$ points. Given a prime $p$, the following hash-function family $H=\left\{h_{a, b}: \mathbb{Z}_{p}^{n} \rightarrow \mathbb{Z}_{p}\right\}$ hashes $n$-vectors and is $(n / p)$-SU. Given $\mathbf{m}=\left(m_{n}, m_{n-1}, \ldots, m_{2}, m_{1}\right)$ with all $m_{i} \in \mathbb{Z}_{p}$ and keys $a, b \in \mathbb{Z}_{p}$, the hash of $\mathbf{m}$ is

$$
h_{a, b}(\mathbf{m})=\left(b+\sum_{i=1}^{n} m_{i} a^{i}\right) \bmod p .
$$


Choosing a random element of $H$ is done by choosing a random $a, b \in \mathbb{Z}_{p}$.

Although this family is $(n / p)$-SU, which is good when $p$ is large and $n$ is not, the hash-function family is not even $(1 / 3)$-VU. Let $n=2$ and $p>3$ be a prime. Let $\mathbf{m}=(0,0), \mathbf{m}^{\prime}=(1,0)$ and $c=0$. Because $n=2$ the hash function is evaluated $h_{a, b}(\mathbf{m})=\left(m_{2} a^{2}+m_{1} a+b\right) \bmod p$. Condition (1) of the $\varepsilon$-VU definition requires $h_{a, b}(\mathbf{m})$ be uniformly distributed over $\mathbb{Z}_{p}$ when $a$ and $b$ are randomly chosen from $\mathbb{Z}_{p}$, which is satisfied because of the random translation $b$. Condition (2) requires computation of the variational distance between the distribution of $h_{a, b}\left(\mathbf{m}^{\prime}\right)$ and $\operatorname{Uniform}\left(\mathbb{Z}_{p}\right)$ when $h_{a, b}(\mathbf{m})=c$. However, because of the values we have selected for $\mathbf{m}, \mathbf{m}^{\prime}$ and $c$, this computation simplifies to $\frac{1}{2} \sum_{y \in \mathbb{Z}_{p}}\left|\operatorname{Pr}_{a \in \mathbb{Z}_{p}}\left[a^{2}=y \bmod p\right]-\frac{1}{p}\right|$, which is exactly $(p-1) / 2 p$, a number greater than $1 / 3$ for any $p>3$.

\section{An Almost-VU Construction}

While SU hash-families are 0-VU, we have already remarked that no SU constructions are known with efficiency comparable to that of best almost-AU constructions. Composing a high-speed almost-AU hash-function family with an SU hash-function family, however, is a good alternative to using an SU family directly. When hashing large inputs, the composite hash-function family will do the bulk of the work in the fast almost-AU part but will be almost-VU because of the subsequent SU component. We now show that this construction works.

Let $A, B$ and $C$ be sets with $B$ and $C$ finite. Let $H=\{h: A \rightarrow B\}$ and $G=\{g: B \rightarrow C\}$ be hash-function families. We define the composed family of functions $G \circ H=\{f: A \rightarrow C\}$ as $\{g \circ h \mid h \in H, g \in G\}$. To choose a random element from $G \circ H$ we choose random elements $h \in H$ and $g \in G$ and consider $f=g \circ h$ to be the random element.

Theorem 1 Let $A, B$ and $C$ be sets with $B$ and $C$ finite. If $H^{\text {au }}=\{h: A \rightarrow B\}$ is $\varepsilon-A U$ and $H^{\mathrm{su}}=\{g: B \rightarrow C\}$ is $S U$ then $H^{\mathrm{su}} \circ H^{\mathrm{au}}$ is $\varepsilon(1-1 /|C|)-V U$.

We note that the theorem's claim is stronger than saying $H^{\mathrm{su}} \circ H^{\mathrm{au}}$ is $\varepsilon$-VU. This is because $\varepsilon=0$ is perfect for $\varepsilon$-VU whereas $\varepsilon=1 /|C|$ is perfect for $\varepsilon$-SU (and $\varepsilon-\mathrm{AU}$ ), and so scaling between the two is inevitable.

PROOF. Let $c \in C$ and let $m, m^{\prime} \in A$ be distinct. For convenience, let $f$ be shorthand for $g \circ h$ and let all probability measures be over the choice of $h \in H^{\text {au }}$ and $g \in H^{\text {su }}$. Because $H^{\text {su }}$ is strongly universal, $g(h(m))$ is uniformly distributed over $C$ for randomly chosen $g \in H^{\mathrm{su}}$ and any value of $h(m)$. 
Let $D$ be the distribution induced on $f\left(m^{\prime}\right)$ when $f$ is chosen randomly and $f(m)=c$. Then, we must show $\operatorname{dist}(D, \operatorname{Uniform}(C)) \leq\left(\varepsilon-\frac{\varepsilon}{|C|}\right)$. We begin by using the definition of dist to rewrite the left-hand side of the desired inequality as

$$
\frac{1}{2} \sum_{y \in C}\left|\operatorname{Pr}\left[f\left(m^{\prime}\right)=y \mid f(m)=c\right]-\frac{1}{|C|}\right| .
$$

Rewrite this expression with the $y=c$ term extracted from the summation,

$$
\frac{1}{2}\left(\left|\operatorname{Pr}\left[f\left(m^{\prime}\right)=c \mid f(m)=c\right]-\frac{1}{|C|}\right|+\sum_{\substack{y \in C \\ y \neq c}}\left|\operatorname{Pr}\left[f\left(m^{\prime}\right)=y \mid f(m)=c\right]-\frac{1}{|C|}\right|\right),
$$

and simplify each of the two halves. First, $\operatorname{Pr}\left[f\left(m^{\prime}\right)=c \mid f(m)=c\right]-1 /|C|$ can be rewritten as

$$
\begin{aligned}
\operatorname{Pr}\left[f\left(m^{\prime}\right)\right. & \left.=c \mid f(m)=c \wedge h\left(m^{\prime}\right)=h(m)\right] \cdot \operatorname{Pr}\left[h\left(m^{\prime}\right)=h(m)\right] \\
+ & \operatorname{Pr}\left[f\left(m^{\prime}\right)=c \mid f(m)=c \wedge h\left(m^{\prime}\right) \neq h(m)\right] \cdot \operatorname{Pr}\left[h\left(m^{\prime}\right) \neq h(m)\right]-\frac{1}{|C|} .
\end{aligned}
$$

Notice that if $h\left(m^{\prime}\right)=h(m)$, then $f\left(m^{\prime}\right)$ must be equal to $f(m)$, and that if $h\left(m^{\prime}\right)$ and $h(m)$ are distinct, then $\operatorname{Pr}\left[f\left(m^{\prime}\right)=f(m)\right]=1 /|C|$ because $H^{\text {su }}$ is strongly universal. Letting $p=\operatorname{Pr}\left[h\left(m^{\prime}\right)=h(m)\right]$, we can simplify Equation 2 to

$$
p+\frac{1}{|C|}(1-p)-\frac{1}{|C|}=p-\frac{p}{|C|} .
$$

Next, we look at the term within the summation in Equation 1, $\operatorname{Pr}\left[f\left(m^{\prime}\right)=\right.$ $y \mid f(m)=c]-\frac{1}{|C|}$, with $y \neq c$, which can be rewritten as

$$
\begin{aligned}
\operatorname{Pr}\left[f\left(m^{\prime}\right)\right. & \left.=y \mid f(m)=c \wedge h\left(m^{\prime}\right)=h(m)\right] \cdot \operatorname{Pr}\left[h\left(m^{\prime}\right)=h(m)\right] \\
+ & \operatorname{Pr}\left[f\left(m^{\prime}\right)=y \mid f(m)=c \wedge h\left(m^{\prime}\right) \neq h(m)\right] \cdot \operatorname{Pr}\left[h\left(m^{\prime}\right) \neq h(m)\right]-\frac{1}{|C|} .
\end{aligned}
$$

This time, because $y \neq c$ and $H^{\text {su }}$ is $\mathrm{SU}, \operatorname{Pr}\left[f\left(m^{\prime}\right)=y \mid f(m)=c \wedge h\left(m^{\prime}\right)=\right.$ $h(m)]=0$ and $\operatorname{Pr}\left[f\left(m^{\prime}\right)=y \mid f(m)=c \wedge h\left(m^{\prime}\right) \neq h(m)\right]=1 /|C|$. Again letting $p=\operatorname{Pr}\left[h\left(m^{\prime}\right)=h(m)\right]$, we can simplify Equation 4 to

$$
0+\frac{1}{|C|}(1-p)-\frac{1}{|C|}=-\frac{p}{|C|}
$$


Substituting Equations 3 and 5 into Equation 1 results in

$$
\frac{1}{2}\left(\left|p-\frac{p}{|C|}\right|+\sum_{\substack{y \in C \\ y \neq c}}\left|-\frac{p}{|C|}\right|\right)=\frac{1}{2}\left(p-\frac{p}{|C|}+(|C|-1) \frac{p}{|C|}\right)=p\left(1-\frac{1}{|C|}\right) .
$$

Finally, $p \leq \varepsilon$ because $p=\operatorname{Pr}\left[h(m)=h\left(m^{\prime}\right)\right]$ and $H^{\text {au }}$ is assumed $\varepsilon$-AU. Thus $\operatorname{dist}(D, \operatorname{Uniform}(C)) \leq \varepsilon(1-1 /|C|)$, as desired.

This construction is a simple way to build a hash-function family that harnesses the speed of fast almost-AU hash families while at the same time providing a stronger guarantee. This same strategy can be applied to accelerate almost-SU hash families too: compose a fast $\varepsilon$-AU hash-function family with an almost-SU hash-function family and you will get a faster almost-SU hashfunction family in return. But for the small price of using an SU family rather than an almost-SU family, the guarantee is more generally useful.

\section{References}

[1] D. Bernstein, The Poly1305-AES message-authentication code, in: Proceedings of Fast Software Encryption, FSE 2005, LNCS vol. 3557, Springer-Verlag, 2005, pp. 32-49.

[2] J. Black, S. Halevi, H. Krawczyk, T. Krovetz, P. Rogaway, UMAC: Fast and secure message authentication, in: Advances in Cryptology - CRYPTO '99, LNCS vol. 1666, Springer-Verlag, 1999, pp. 216-233.

[3] L. Carter, M. Wegman, Universal classes of hash functions, J. of Computer and System Sciences 18 (1979), pp. 143-154.

[4] S. Halevi, H. Krawczyk, MMH: Software message authentication in the Gbit/second rates, in: Proceedings of Fast Software Encryption, FSE 1997, LNCS vol. 1267, Springer-Verlag, 1997, pp. 172-189

[5] V. Shoup, On fast and provably secure message authentication based on universal hashing, in: Advances in Cryptology - CRYPTO '96, LNCS vol. 1109, SpringerVerlag, 1996, pp. 313-328.

[6] D. Stinson, Universal hashing and authentication codes, Designs, Codes and Cryptography 4 (1994), pp. 369-380.

[7] M. Wegman, L. Carter, New hash functions and their use in authentication and set equality, J. of Computer and System Sciences 22 (1981), pp. 265-279. 